\title{
Partial Component Consensus of Discrete-Time Multiagent Systems
}

\author{
Wenjun Hu, ${ }^{1,2}$ Gang Zhang $\left(D,{ }^{1}\right.$ Zhongjun $M a\left(D,{ }^{3}\right.$ and Binbin $\mathrm{Wu}^{3}$ \\ ${ }^{1}$ College of Mathematics and Information Science, Hebei Normal University, Shijiazhuang 050024, China \\ ${ }^{2}$ Department of Mathematics, Luliang University, Lishi 033000, China \\ ${ }^{3}$ College of Mathematics and Computing Science, Guilin University of Electronic Technology, Guilin 541004, China
}

Correspondence should be addressed to Gang Zhang; gangzhang@hebtu.edu.cn

Received 21 August 2018; Accepted 4 April 2019; Published 16 April 2019

Academic Editor: Zhiyun Lin

Copyright (C) 2019 Wenjun Hu et al. This is an open access article distributed under the Creative Commons Attribution License, which permits unrestricted use, distribution, and reproduction in any medium, provided the original work is properly cited.

\begin{abstract}
The multiagent system has the advantages of simple structure, strong function, and cost saving, which has received wide attention from different fields. Consensus is the most basic problem in multiagent systems. In this paper, firstly, the problem of partial component consensus in the first-order linear discrete-time multiagent systems with the directed network topology is discussed. Via designing an appropriate pinning control protocol, the corresponding error system is analyzed by using the matrix theory and the partial stability theory. Secondly, a sufficient condition is given to realize partial component consensus in multiagent systems. Finally, the numerical simulations are given to illustrate the theoretical results.
\end{abstract}

\section{Introduction}

In recent years, the theory of consistency, as the basis of coordinated control of multiagent system, has attracted extensive attention from many researchers [1-3]. The unified nature of multiagent systems is wildly applied to computing science [4], systems and control [5-7], and distributed sensor networks [8-10].

The consistency of the discrete multiagent systems is that the state of all agents in a discrete system model can achieve asymptotic convergence under certain conditions. Many researchers have discussed the consistency problem of multiagent systems [11-14] and have obtained a lot of research results. Xie Dongmei and Wang Shaokun considered the consensus of second-order discrete-time multiagent systems with fixed topology in [15]. In 2016, Gao Yulan et al. studied group consensus for second-order discrete-time multiagent systems with time-varying delays under switching topologies in [16]. At the same year, Cao Yanfen and Sun Yuangong discussed consensus of discrete-time third-order multiagent systems in directed networks in [17]. Furthermore, the consensus of leader-following multiagent has also received a lot of attention. Wang Yunpeng et al. proposed an algorithm to research the consensus of discrete-time linear multiagent systems with communication noises in [18]. Xu Xiaole et al. investigated the leader-following consensus problem of discrete-time multiagent systems through Lyapunov method in [19].

The consistency of discrete multiagent systems has more advantages than continuous multiagent systems. For example, it can reduce a lot of computation, and the speed of convergence is fast and so on. Therefore, the research of discrete consistency has some practical significance. Wu Binbin et al. have studied the partial component consensus of continuous multiagent systems in [20]. In this paper, we discuss the partial component consistency of discrete leaderfollowing multiagent system. Based on the matrix theory and the partial stability theory, together with designing an appropriate pinning control protocol, a sufficient condition is proposed to realize partial component consensus in multiagent systems.

In detail, the remainder of this paper is organized as follows: Section 2 contains the problem statement and preliminaries; Section 3 presents the main result about the partial component consistency of discrete leader-following multiagent system; Section 4 provides a numerical example 
to verify the effectiveness of the proposed results; Section 5 offers concluding remarks.

\section{The Problem Statement and Preliminaries}

In this section, we will give the basic concept of partial component consistency, basic matrix theory, and some definitions and lemmas. For details, refer to [20, 21].

We consider the following $\mathrm{n}$-dimensional discrete system:

$$
x(k+1)=F(x(k)),
$$

where $F(x) \in C\left[R^{n}, R^{n}\right], F(0) \equiv 0, x=\left(x_{1}, \ldots, x_{m}, x_{m+1}\right.$, $\left.\ldots, x_{n}\right) \in R$. Supposing $y=\left(x_{1}, \ldots, x_{m}\right), z=\left(x_{m+1}, \ldots, x_{n}\right)$, $(m+p=n)$, and $\|x\|=\left(\sum_{i=1}^{n} x_{i}^{2}\right)^{1 / 2},\|y\|=\left(\sum_{i=1}^{m} x_{i}^{2}\right)^{1 / 2}$, $\|z\|=\left(\sum_{i=m+1}^{n} x_{i}^{2}\right)^{1 / 2}, k \in N$.

Similar to the definition of partial component stability for continuous system in [21], we give the following definition of partial component stability for discrete system.

Definition 1. The trivial solution of (1) is stable for vector $y$, if $\forall \varepsilon>0, \forall k_{0} \in N, \exists \delta\left(k_{0}\right)>0, \forall x_{0} \in S_{\delta\left(k_{0}, \varepsilon\right)}=x \|<\delta\left(k_{0}, \varepsilon\right)$, and when $k \geq k_{0}$, there must be $\left\|y\left(k, k_{0}, x_{0}\right)\right\|<\varepsilon$.

Definition 2. The trivial solution of (1) is attracted to vector $y$, if $\forall k_{0} \in N, \exists \delta\left(k_{0}\right)>0, \forall \varepsilon>0, \forall x_{0} \in S_{\sigma\left(k_{0}\right)}=x\|x\| \leq$ $\delta\left(k_{0}\right), \exists T\left(k_{0}, x_{0}, \varepsilon\right)>0$, and when $t \geq k_{0}+T$, there must be $\left\|y\left(k, k_{0}, x_{0}\right)\right\|<\varepsilon$, where $S_{\sigma\left(k_{0}\right)}$ is the attraction area of the vector $y$.

Definition 3. The trivial solution of (1) is asymptotically stable for vector $y$, if it is stable and attracted for vector $y$.

Lemma 4 (see [20]). Let $H=\left(h_{i j}\right) \in R^{N \times N}, B=\left(b_{i j}\right) \in R^{n \times n}$, then there exists a $n N$-order permutation matrix $P=P_{1} \cdots P_{s}$, where $P_{i}$ is the first kind of elementary row transformation matrix, such that the $P(H \otimes B) P^{-1}=B \otimes H$ can hold, where $i=1, \ldots, s, s \in N_{+}, \otimes$ is the Kronecker product.

\section{Main Results}

In this section, we consider a first-order discrete multiagent system, which consists of $\mathrm{N}$ following agents and a leader, and suppose the equation of state for the following agent is

$$
x_{i}(k+1)=A x_{i}(k)+u_{i}(k), \quad i=1,2, \ldots, N,
$$

where, $x_{i}(k)=\left(x_{i 1}(k), \ldots, x_{i n}(k)\right)^{T} \in R^{n}$ expresses the state of ith agent; $A=\operatorname{diag}\left(A_{1}, \ldots, A_{n}\right)$ is a diagonal matrix.

Supposing the dynamic equation of the leader agent is

$$
x_{0}(k+1)=A x_{0}(k)
$$

where $x_{0}(k)=\left(x_{01}(k), \ldots, x_{0 n}(k)\right)^{T} \in R^{n}$ is the state of leader agent.

Next we consider the problem of partial component conformance of discrete leader-following multiagent system under directed network topology. We design the controller as follows:

$$
\begin{aligned}
u_{i}(k)= & c \sum_{j=1}^{N} a_{i j} \Gamma\left(x_{j}(k)-x_{i}(k)\right) \\
& -c d_{i} \Gamma\left(x_{i}(k)-x_{0}(k)\right),
\end{aligned}
$$

where $c>0$ denotes coupling strength; $\Gamma=\operatorname{diag}\left(r_{1}, \ldots, r_{n}\right) \in$ $R^{n \times n}\left(r_{k} \geq 0, k=1, \ldots, n\right)$ is the intercoupling matrix; if the ith agent can receive the information from the $j t h$ agent, then $a_{i j}>0$; otherwise, $a_{i j}=0$. If the ith agent can receive the information from the leader agent, then $d_{i}>0$; otherwise, $d_{i}>0$.

Let $L=\left(L_{i j}\right)$ satisfy $L_{i j}=-s_{i j}(i \neq j)$ and $L_{i i}=\sum_{i \neq j}$, and let $e_{i}(k)=x_{i}(k)-x_{0}(k)$. We can get the error system from (2), (3), and (4) as follows:

$$
e_{i}(k+1)=A e_{i}(k)-c(L+D) \otimes \Gamma e_{i}(k) .
$$

We can write the above equation as a vector form:

$$
e(k+1)=\left[I_{N} \otimes A I_{n}-c(L+D) \otimes \Gamma\right] e(k) .
$$

where $e(k)=\left(e_{1}(k), \ldots, e_{N}(k)\right)^{T}, e_{i}(k)=\left(e_{i 1}(k), \ldots, e_{i n}(k)\right)^{T}$, $D=\operatorname{diag}\left(d_{1}, \ldots, d_{N}\right), I_{n}$ represents n-order identity matrix.

In order to discuss the asymptotic stability of the trivial solution of the error system (6), we try to do the following transformation. Let $\widehat{e}(k+1)=P e(k)$ and $\varepsilon_{j}(k)=$ $\left(e_{1 j}(k), \ldots, e_{N j}(k)\right)$, where $P$ is the elementary matrix in Lemma 4 and $j=1, \ldots, n$. We can get $\widehat{e}(k)=\left(\varepsilon_{1}^{T}(k), \ldots\right.$, $\left.\varepsilon_{n}^{T}(k)\right)^{T}$ through calculation; in this case, (6) will change to the following equation:

$$
\widehat{e}(k+1)=P\left[I_{N} \otimes A I_{n}-c(L+D) \otimes \Gamma\right] P^{-1} \widehat{e}(k) .
$$

We can simplify the above equation,

$$
\widehat{e}(k+1)=\left[A I_{n} \otimes I_{N}-c \Gamma \otimes(L+D)\right] \widehat{e}(k) .
$$

Next we give two useful definitions for this paper.

Definition 5. If there exist $1 \leq l \leq n$, such that the solution of system (2) and (3) satisfy $\lim _{k \rightarrow \infty} \sum_{j=1}^{l}\left\|\varepsilon_{j}(k)\right\|=0$, then system (2) and (3) achieve consensus for the first $l$ components.

Definition 6. If there exist $l=1$, such that the solution of system (2) and (3) satisfy $\lim _{k \rightarrow \infty}\left\|\varepsilon_{1}(k)\right\|=0$, then system (2) and (3) achieve consensus for the first component.

Theorem 7. Let $M_{j}=A_{j} I_{N}-c r_{j}(L+D), N_{j}=M_{j}^{T} M_{j}$, for the control item, if there exist $\beta \in(0,1)$, such that system (2) and (3) satisfy

$$
N_{j}<-(\beta-1) I_{N}, \quad j=1,2, \ldots, l,
$$

where $r_{j}>0$ is the jth element of the matrix $\Gamma=$ $\operatorname{diag}\left(r_{1}, \ldots, r_{n}\right), D=\operatorname{diag}\left(d_{1}, \ldots, d_{N}\right), d_{i} \geq 0$; then, system (2) and (3) can achieve consensus for the first l components. 
Proof. Define the following Lyapunov function candidate:

$$
V(k)=\sum_{j=1}^{l} \sum_{i=1}^{N} e_{i k}^{T}(k) e_{i k}(k)=\widehat{e}^{T}(k) \wedge \widehat{e}(k),
$$

where $\wedge=\operatorname{diag}(\overbrace{1, \ldots, 1}^{l N}, 0, \ldots, 0), \widehat{e}(k)=\left(\varepsilon_{1}^{T}(k), \ldots, \varepsilon_{n}^{T}(k)\right)^{T}$. Similar to literature [19], we can obtain the following:

$$
\begin{aligned}
& \Delta V(k)=V(k+1)-V(k) \\
& =\hat{e}^{T}(k)\{[A \\
& \left.\left.\otimes I_{N}-c \Gamma \otimes(L+D)\right]^{T} \wedge\left[A \otimes I_{N}-c \Gamma \otimes(L+D)\right]\right\} \widehat{e}(k)-\widehat{e}^{T}(k) \wedge \widehat{e}(k) \\
& =\sum \widehat{\varepsilon}_{j}^{T}(k)\left\{\left[A_{j} I_{N}-c r_{j}(L\right.\right. \\
& \left.+D)]^{T}\left[A_{j} I_{N}-c r_{j}(L+D)\right]-I_{N}\right\} \widehat{\varepsilon}_{j}(k) \\
& =\sum \widehat{\varepsilon}_{j}^{T}(k)\left(M_{j}^{T} M_{j}\right. \\
& \left.-I_{N}\right) \widehat{\varepsilon}_{j}(k) \\
& =\widehat{e}^{T}(t)
\end{aligned}
$$

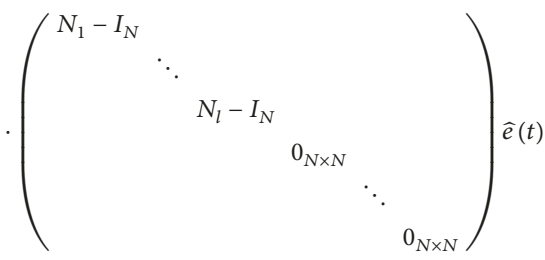

$$
\begin{aligned}
& \leq-\sum_{j=1}^{l} \beta \varepsilon_{j}^{T}(k) \varepsilon_{j}(k)=-\beta V(k) .
\end{aligned}
$$

In this case, if (9) can be held, then there exist $\beta \in(0,1)$, such that

$$
\begin{aligned}
\Delta V(k) & =V(k+1)-V(k) \leq-\sum_{j=1}^{l} \beta \varepsilon_{j}^{T}(k) \varepsilon_{j}(k) \\
& =-\beta V(k) .
\end{aligned}
$$

Therefore,

$$
\begin{aligned}
V(k+1) & \leq V(k)-\beta V(k)=(1-\beta)^{2} \\
& \leq(1-\beta)^{2} V(k-1) \leq \cdots \\
& \leq(1-\beta)^{k+1} V(0),
\end{aligned}
$$

when $k \longrightarrow \infty, V(k+1) \leq(1-\beta)^{k+1} V(0)$, i.e., $\lim _{k \rightarrow \infty} \varepsilon_{j}(k)=$ 0 . Therefore, $\lim _{k \rightarrow \infty} \sum_{j=1}^{l}\left\|\varepsilon_{j}(k)\right\|=0$; then the solution of (8) is asymptotically stable for partial vector element; i.e., system (2) and (3) can achieve consensus for the first $l$ components.

\section{Numerical Examples}

In this section, a numerical example has been given to show that our theoretical result obtained above is effective.

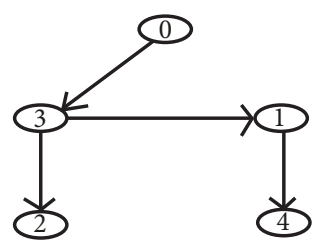

FIGURE 1: The topology diagram of agent connection.

Example 1. Given the parameter $n$ and $N$ in system (2) as $n=$ 3 and $N=4$, we consider the consensus of the discrete leaderfollowing multiagent system for the first two components (i.e., $l=2$ ). Supposing the state of the $i t h$ agent (the subscript of the state of the leader denotes 0$)$ is $x_{i}(k+1)=A x_{i}(k)$, $A=\operatorname{diag}(1.01,1.01,1.03)$. Designing $L$ and $D$ as follows:

$$
\begin{aligned}
L & =\left(\begin{array}{cccc}
1 & 0 & -1 & 0 \\
0 & 1 & -1 & 0 \\
0 & 0 & 0 & 0 \\
-1 & 0 & 0 & 1
\end{array}\right), \\
D & =\left(\begin{array}{llll}
0 & 0 & 0 & 0 \\
0 & 0 & 0 & 0 \\
0 & 0 & 1 & 0 \\
0 & 0 & 0 & 0
\end{array}\right),
\end{aligned}
$$

then bring $A, L$, and $D$ into (4). Through simple calculations, at the same time, we set $c=0.3$ and $\Gamma=\operatorname{diag}(1,1,0)$; then, (9) will be held. In this case, it is straightforward to check that all the conditions in Theorem 7 hold. Next we will give the topology diagram of agent connection and the error trajectories of system (2) and (3) through Matlab software.

Figure 1 expresses the topology connection of the given agent. Next we give the evolution diagram of the state error of the leader-following agent (Figure 2); Figures 2(a) and 2(b) represent the consensus of system (2) and (3) for the first two components and Figure 2(c) represents that system (2) and (3) cannot achieve consensus for the third component.

\section{Conclusion}

In this paper, the partial component consensus has been investigated for the first-order discrete leader-following multiagent system. By establishing the suitable control term and using the matrix theory together with the stability theory, the sufficient conditions for the partial component conformance of the discrete system are derived. Furthermore, a numerical example has been given to illustrate the effectiveness of the present results. As an extension to this work, we plan to discuss the partial component consensus for the high-order discrete leader-following multiagent system.

\section{Data Availability}

No data were used to support this study. 


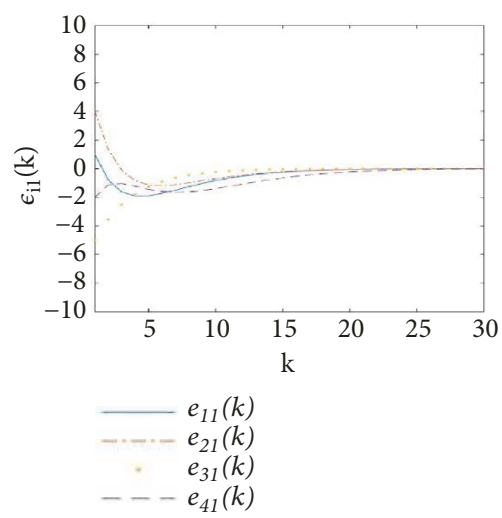

(a)

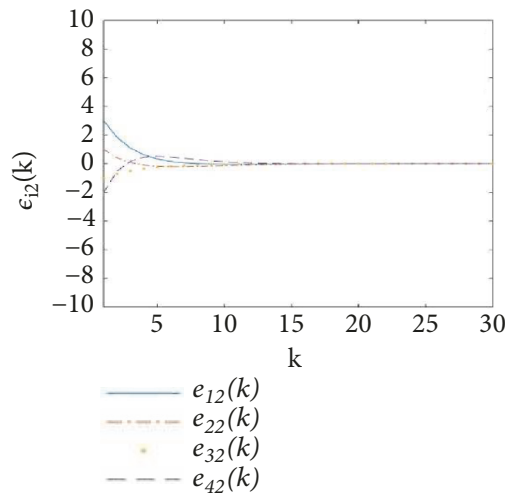

(b)

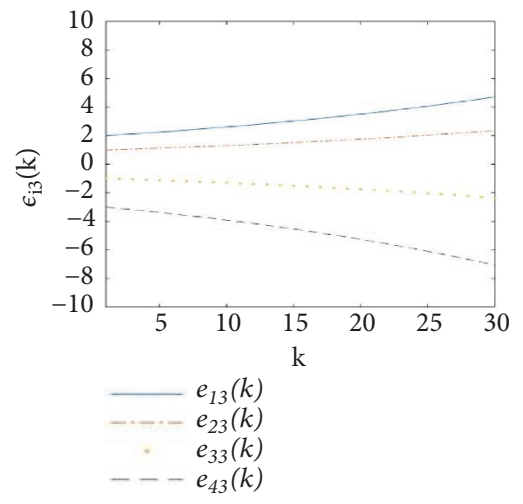

(c)

Figure 2: (a) The state error of the leader-following for the first component. (b) The state error of the leader-following for the second component. (c) The state error of the leader-following for the third component.

\section{Conflicts of Interest}

The authors declare that they have no conflicts of interest.

\section{Acknowledgments}

The authors gratefully acknowledge the support of the National Natural Science Foundation of China (NNSF) through Grant (No. 11562006), the NSF of Guangxi Province (2018GXNSFAA281068), and the NSF of Shanxi Province (201801D121009).

\section{References}

[1] T. Vicsek, A. Czirk, E. Ben-Jacob, I. Cohen, and O. Shochet, "Novel type of phase transition in a system of self-driven particles," Physical Review Letters, vol. 75, no. 6, pp. 1226-1229, 1995.

[2] A. Jadbabaie, J. Lin, and A. S. Morse, "Coordination of groups of mobile autonomous agents using nearest neighbor rules," IEEE Transactions on Automatic Control, vol. 48, no. 6, pp. 988-1001, 2003.

[3] R. Olfati-Saber and R. M. Murray, "Consensus problems in networks of agents with switching topology and time-delays," IEEE Transactions on Automatic Control, vol. 49, no. 9, pp. 15201533, 2004.

[4] G. Wen, Z. Duan, W. Yu, and G. Chen, "Consensus of multiagent systems with nonlinear dynamics and sampled-data information: a delayed-input approach," International Journal of Robust and Nonlinear Control, vol. 23, no. 6, pp. 602-619, 2013.

[5] W. Ren, R. W. Beard, and T. W. McLain, "Coordination variables and consensus building in multiple vehicle systems," in Cooperative Control, vol. 309 of Lect. Notes Control Inf. Sci., pp. 171-188, Springer, Berlin, Germany, 2005.

[6] Y. Hong, J. Hu, and L. Gao, "Tracking control for multiagent consensus with an active leader and variable topology," Automatica, vol. 42, no. 7, pp. 1177-1182, 2006.

[7] J. Mei, W. Ren, and G. Ma, "Distributed coordinated tracking with a dynamic leader for multiple Euler-Lagrange systems," IEEE Transactions on Automatic Control, vol. 56, no. 6, pp. 14151421, 2011.
[8] N. A. Lynch, Distributed Algorithms, Morgan Kaufman Publisher, San Francisco, Calif, USA, 1996.

[9] J. N. Tsitsiklis and M. Athans, "Convergence and asymptotic agreement in distributed decision problems," IEEE Transactions on Automatic Control, vol. 29, no. 1, pp. 42-50, 1984.

[10] Y. Wang, Z. Wang, J. Liang, Y. Li, and M. Du, "Synchronization of stochastic genetic oscillator networks with time-delays and markovian jumping parameters," Neurocomputing, vol. 73, no. 13-15, pp. 2532-2539, 2010.

[11] W. Ren and R. W. Beard, "Consensus seeking in multi-agent systems under dynamically changing interaction topologies," IEEE Transactions on Automatic Control, vol. 50, no. 5, pp. 655661, 2005.

[12] Y. Shang, "Continuous-time average consensus under dynamically changing topologies and multiple time-varying delays," Applied Mathematics and Computation, vol. 244, no. 2, pp. 457466, 2014.

[13] F. Xiao and L. Wang, "Consensus protocols for discrete-time multi-agent systems with time-varying delays," Automatica, vol. 44, no. 10, pp. 2577-2582, 2008.

[14] D. Xie, Q. Liu, L. Lv, and S. Li, "Necessary and sufficient condition for the group consensus of multi-agent systems," Applied Mathematics and Computation, vol. 243, pp. 870-878, 2014.

[15] D. M. Xie and S. K. Wang, "Consensus of second-order discrete-time multi-agent systems with fixed topology," Journal of Mathematical Analysis and Applications, vol. 387, no. 1, pp. 816, 2012.

[16] Y. L. Gao, J. Y. Yu, J. L. Shao, and M. Yu, "Group consensus for second-order discrete-time multi-agent systems with timevarying delays under switching topoloies," Neurocomputing, vol. 207, pp. 805-812, 2016.

[17] Y. Cao and Y. Sun, "Consensus of discrete-time third-order multi-agent systems in directed networks," Neurocomputing, vol. 177, pp. 394-400, 2016.

[18] Y. P. Wang, L. Cheng, H. M. Wang, Z. G. Hou, M. Tan, and N. H. Yu, "Leader-follow consensus of discrete-time linear multi-agent systems with communication noises," in Proceeding of the 34th Chinese Control Conference Hangzhou, pp. 28-30, Hangzhou, China, 2015. 
[19] X. Xu, S. Chen, W. Huang, and L. Gao, "Leader-following consensus of discrete-time multi-agent systems with observerbased protocols," Neurocomputing, vol. 118, no. 22, pp. 334-341, 2013.

[20] B. B. Wu, Z. J. Ma, and Y. Wang, "Partial component consensus of leader-following multi-agent systems," Journal of Physics, vol. 66, no. 6, Article ID 060201, 2017.

[21] X. X. Liao, The Stability of Mathematical Theory and Its Application, Central China Normal University Publisher, 2001. 


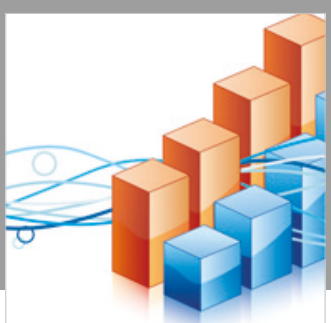

Advances in

Operations Research

\section{-n-m}
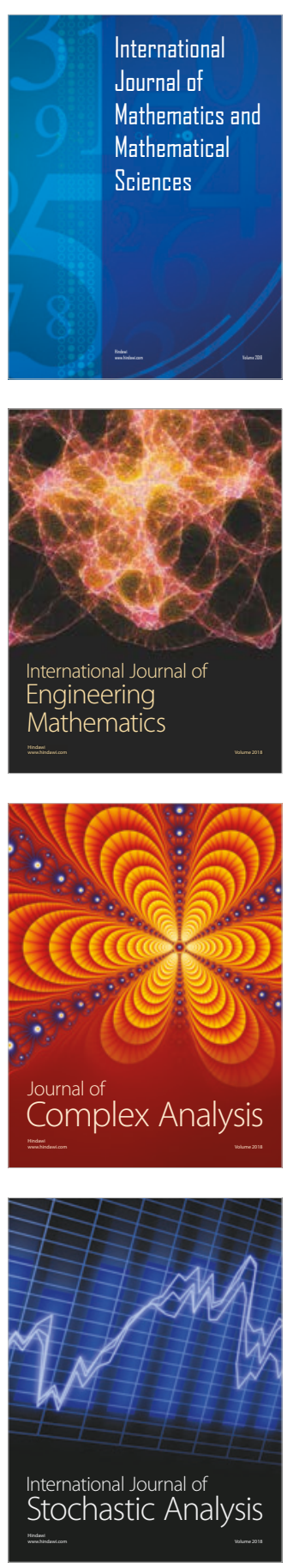
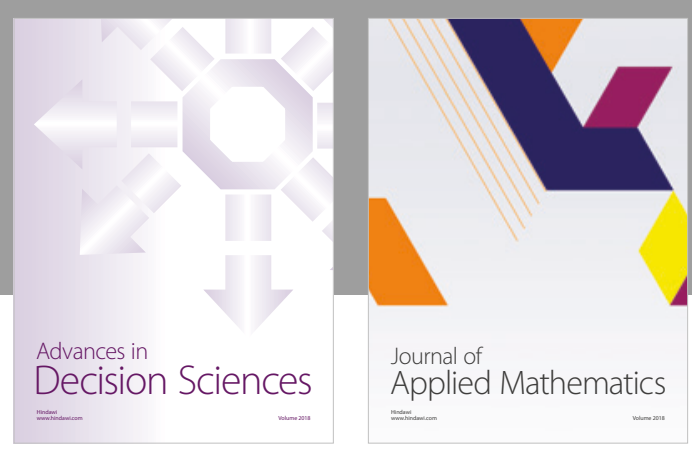

Journal of

Applied Mathematics
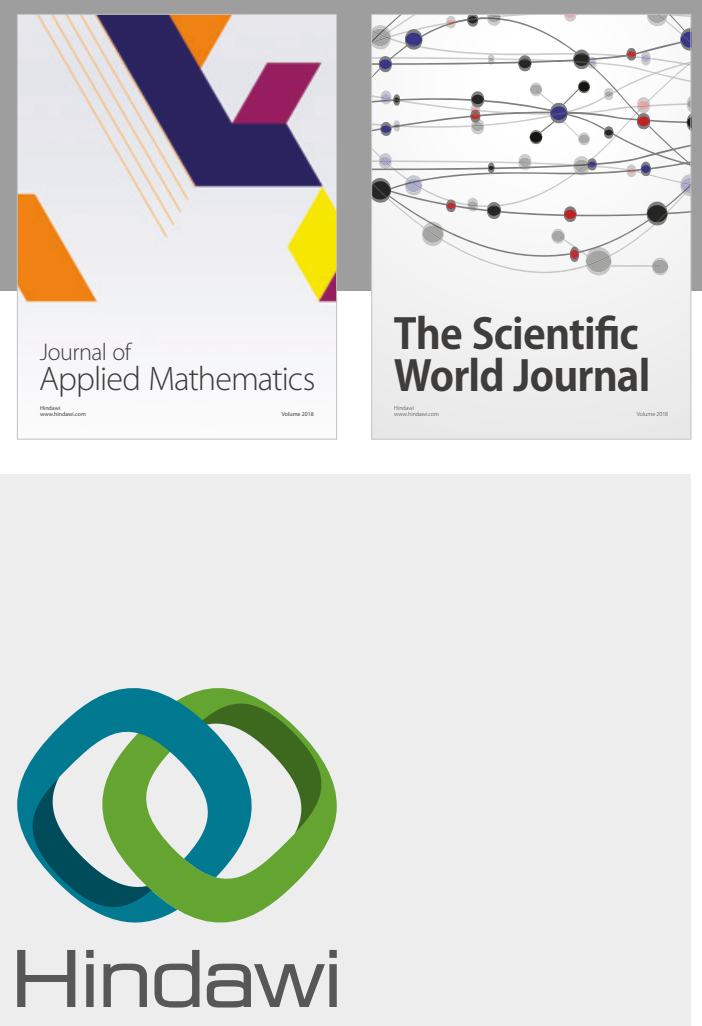

Submit your manuscripts at

www.hindawi.com

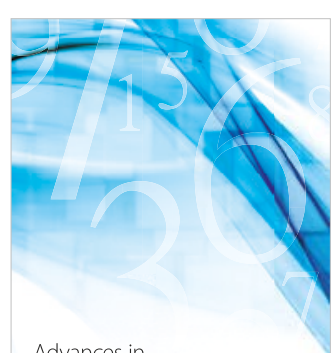

Advances in
Numerical Analysis
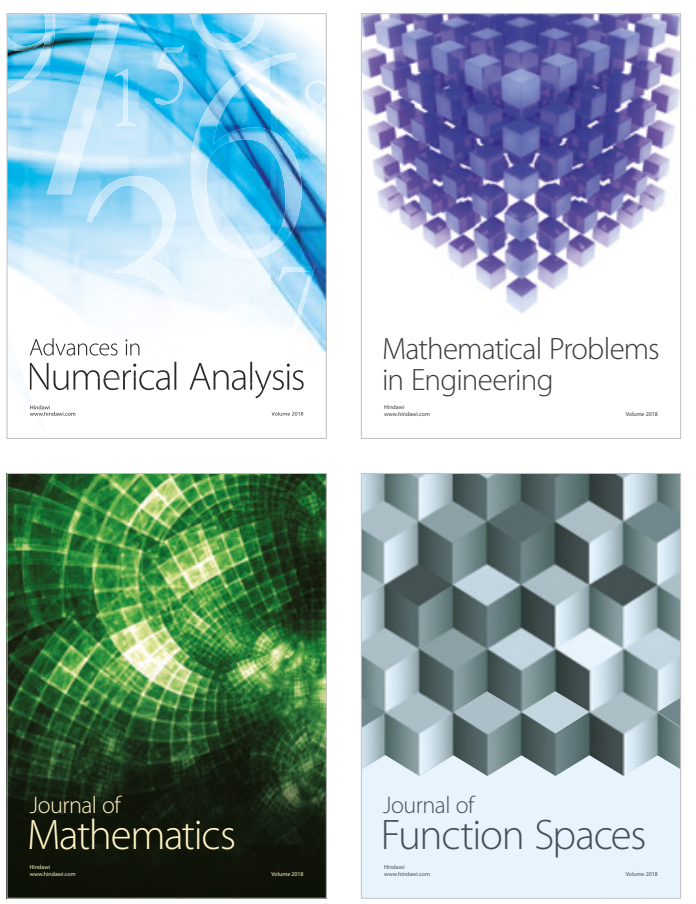

Mathematical Problems in Engineering

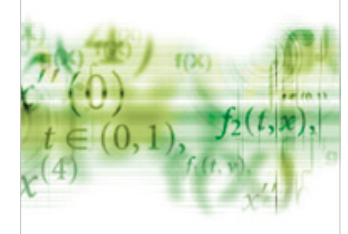

International Journal of

Differential Equations

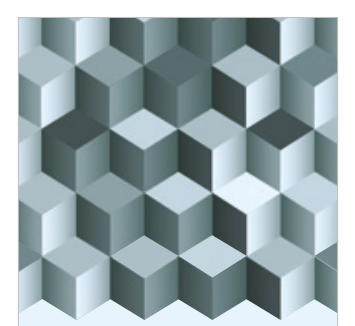

Journal of

Function Spaces

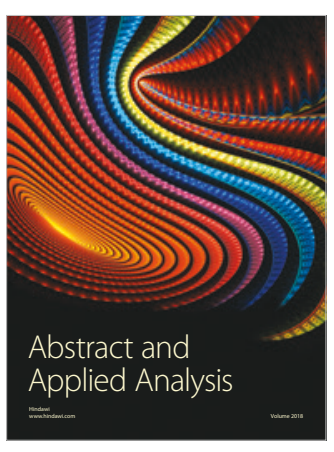

The Scientific

World Journal

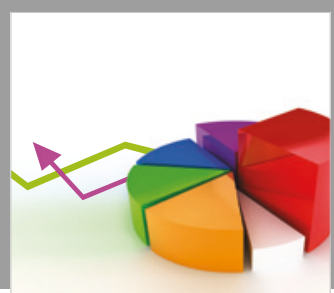

Journal of

Probability and Statistics
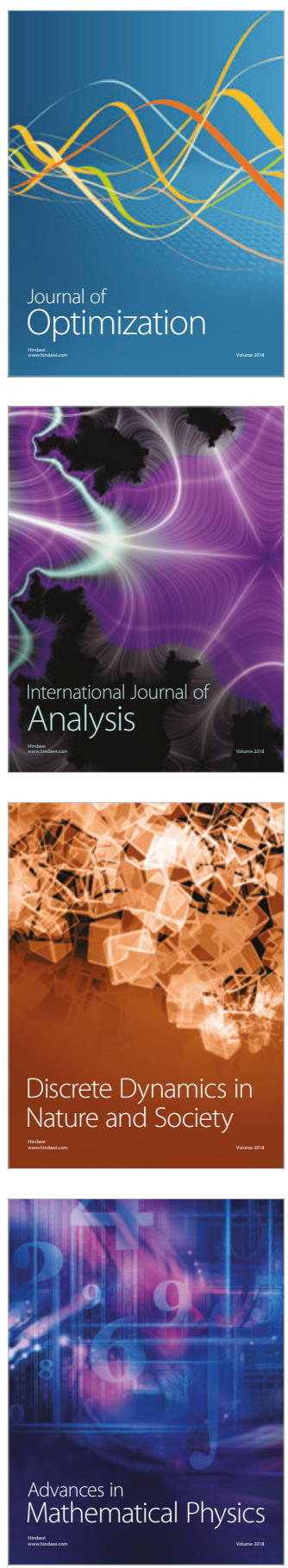